\title{
Editorial
}

\section{Fiber Optic Sensors}

\author{
Wolfgang Ecke, ${ }^{1}$ Kevin Chen, ${ }^{2}$ and Jinsong Leng ${ }^{3}$ \\ ${ }^{1}$ Department of Fiber Optics, Institute of Photonic Technology, 07745 Jena, Germany \\ ${ }^{2}$ Department of Electrical and Computer Engineering, Swanson School of Engineering, Pittsburgh University, \\ Pittsburgh, PA 15261, USA \\ ${ }^{3}$ Center of Composite Materials and Structures, Harbin Institute of Technology, Harbin 150080, China
}

Correspondence should be addressed to Wolfgang Ecke, ecke@ipht-jena.de

Received 12 June 2012; Accepted 12 June 2012

Copyright (c) 2012 Wolfgang Ecke et al. This is an open access article distributed under the Creative Commons Attribution License, which permits unrestricted use, distribution, and reproduction in any medium, provided the original work is properly cited.

Optical fiber sensors find increasing applications in areas ranging from structural health monitoring to biophotonic sensing. The unique properties of optical fibers and sensor structures support such progress: complete immunity to electromagnetic fields, high voltage, lightning; operation in explosive or chemically aggressive and corrosive media (energy, oil, gas); light weight, miniaturized, flexible; low thermal conductivity, temperature-resistant material (highend and low-end temperatures); low-loss, noninterfering signal transmission, ability to operate over long distances (remote sensing), multiplexing capability (sensor networks), and structure monitoring by embedding in composite materials (smart structures).

New optical wave-guide materials and systems like plastic optical fibers, sapphire optical fiber, photonic crystal fibers, and other nanostructures stimulate corresponding new sensor developments making use of the specific material advantages.

In this issue you will find some examples of actual research, scientific technological development, and application of optical fiber sensors: a review on the status of photonic crystal fibers for sensing applications; fiber Bragg grating resonator structures resulting in an all-fiber DBRbased sensor interrogation system for measuring acoustic waves; the application of plastic optical fibers for sensing of fuel leakage in soil as well as of alcohol concentration in liquors; application of laser Doppler velocimetry for measurement of local blood velocity profiles.

Wolfgang Ecke

Kevin Chen

Jinsong Leng 

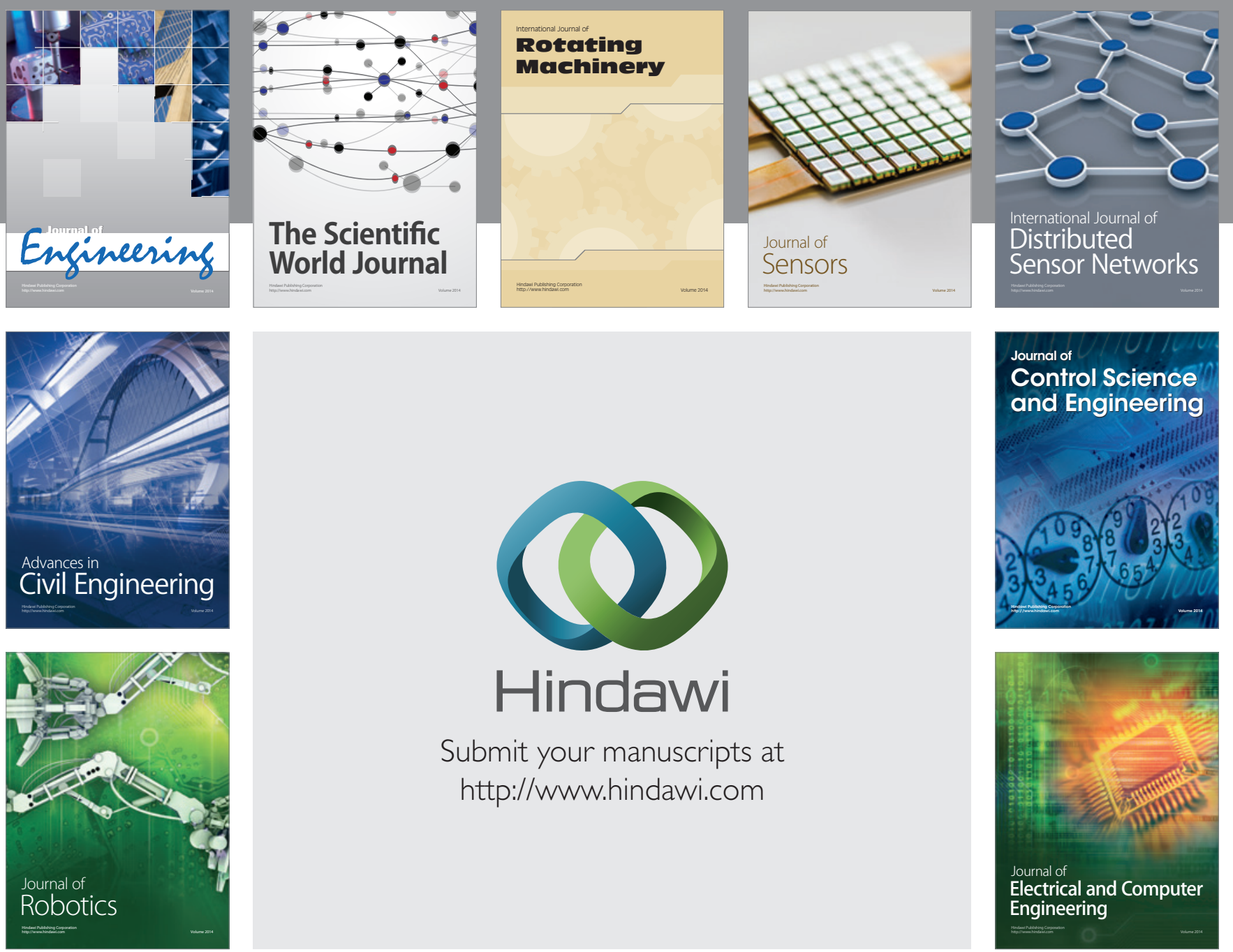

Submit your manuscripts at

http://www.hindawi.com
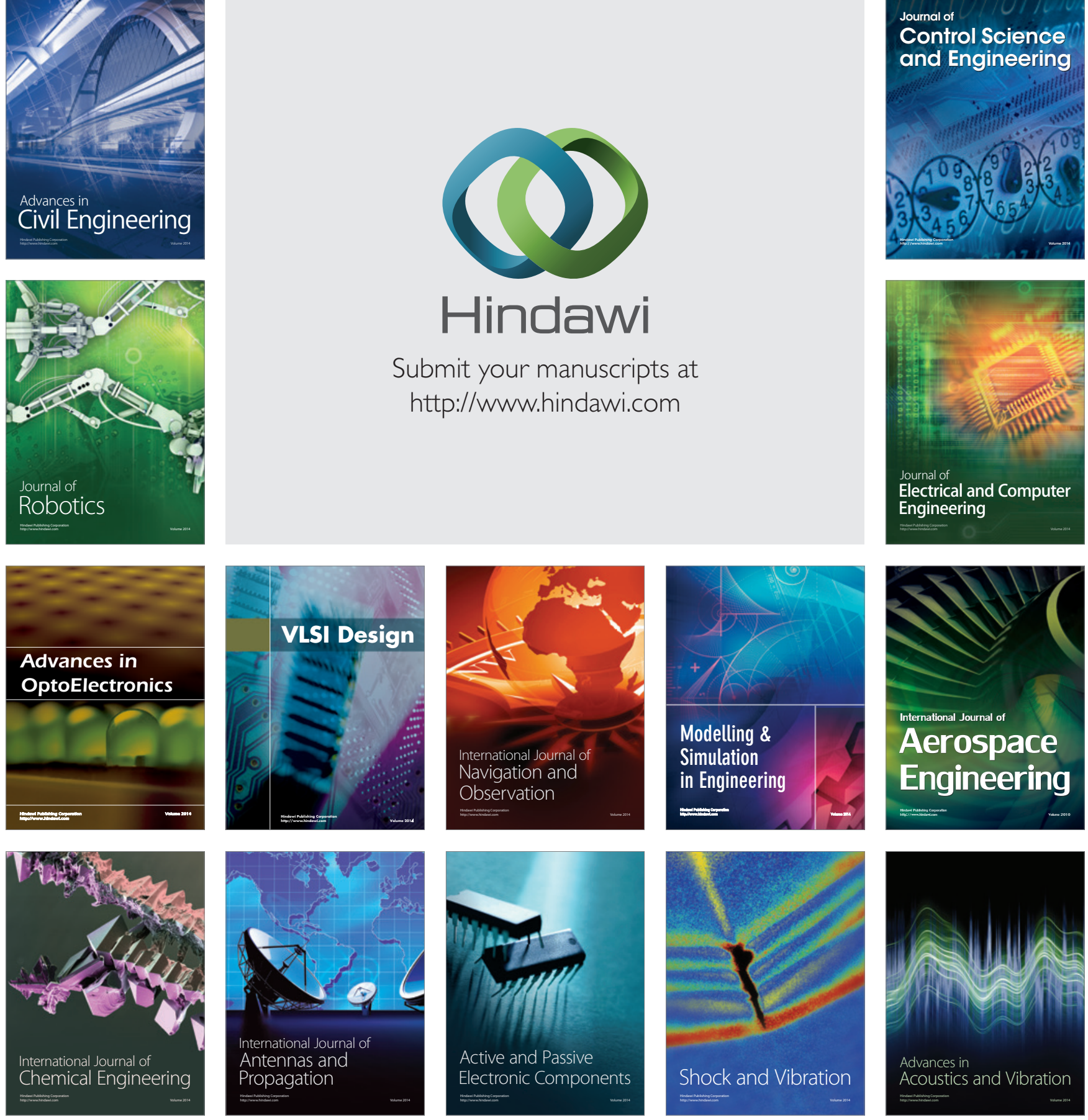